\title{
Continuity bounds for entanglement
}

\author{
M. A. Nielsen* \\ Physics MC 12-33, California Institute of Technology, Pasadena, California 91125
}

(Received 3 September 1999; published 28 April 2000)

\begin{abstract}
This Brief Report quantifies the continuity properties of entanglement: how much does entanglement vary if we change the entangled quantum state just a little? This question is studied for the pure state entanglement of a bipartite system and for the entanglement of formation of a bipartite system in a mixed state.

PACS number(s): 03.67.- a, 03.65.Bz
\end{abstract}

Entanglement is a resource at the heart of quantum mechanics; iron in the classical world's bronze age. Entanglement plays a crucial role in diverse quantum effects such as Bell inequalities [1], quantum algorithms [2,3], quantum teleportation [4], and paradoxically is also responsible for the emergence of a classical world out of the quantum [5].

To flesh out the notion that entanglement is a resource, various measures of entanglement have been proposed to quantify the amount of entanglement shared between two or more quantum systems. For a pure state of a two-party quantum system, Popescu and Rohrlich [6] and Vidal [7] showed that the measure of entanglement is uniquely specified by certain natural axioms: ${ }^{1}$ it is given by the von Neumann entropy of the reduced density matrix associated with one of the parties. That is, if $|\psi\rangle$ is the state of a composite system with components $A$ and $B$, then the pure state entanglement of $|\psi\rangle$ is given by $E(\psi)=S\left(\rho_{A}\right)=S\left(\rho_{B}\right)$, where $\rho_{A}$ $\equiv \operatorname{tr}_{B}(|\psi\rangle\langle\psi|)$ and $\rho_{B} \equiv \operatorname{tr}_{A}(|\psi\rangle\langle\psi|)$ are the reduced density matrices of system $A$ and system $B$, respectively, and $S(\cdot)$ is the von Neumann entropy.

The situation for mixed-state entanglement is more complex, and a plethora of measures have been developed (see Refs. [8-11,7] and other references cited therein). Perhaps the best understood of these measures is the entanglement of formation studied in a series of papers by Wootters and coworkers [8-10]. For pure states the entanglement of formation reduces to the von Neumann entropy of the reduced density matrix, as expected. However, for mixed states the entanglement of formation shows much more complex behavior, a behavior that is not yet fully understood.

This Brief Report develops inequalities expressing continuity properties of the pure-state entanglement and the entanglement of formation. We begin with the simple arguments needed to prove such results for the pure-state entanglement. This allows us to introduce some of the tools needed for the more complex argument for entanglement of formation, and also gives more stringent bounds than in the mixed-state case. We will show that, up to constants, the bounds are optimal with respect to the dimension of the underlying Hilbert space. Furthermore, we explicitly show that the continuity bounds obtained for the entanglement of for-

\footnotetext{
*Electronic address: mnielsen@theory.caltech.edu

${ }^{1}$ It is to be emphasized that these axioms are only natural if one considers manipulations of large blocks of identically entangled states [7].
}

mation and pure-state entanglement are stronger than those obtainable for other entanglement monotones, continuing a line of thought initiated by Vidal [7]. The continuity bounds we obtain can be applied to analyze approximate schemes for quantum communication protocols, quantum cloning, and quantum communication complexity, work that will be presented elsewhere. The continuity of pure state entanglement has been previously noted by Horodecki et al. [12], although explicit bounds on its variation were not given.

To understand how the entanglement between systems $A$ and $B$ varies as we vary the density matrix for the combined system, we need to introduce some distance measures on density matrices. We will make use of three closely related distance measures in our work: the trace distance, the fidelity, and the Bures distance. To begin we need only the trace distance. The trace distance between density matrices $\rho$ and $\sigma$ is defined to be $T(\rho, \sigma) \equiv \operatorname{tr}|\rho-\sigma|$, where we define $|A|$ $\equiv \sqrt{A^{\dagger} A}$ to be the positive square root of $A^{\dagger} A$. It is easy to see that the trace distance is a metric on the space of density matrices. Furthermore, Ruskai [13] showed that the trace distance is nonincreasing under quantum operations. That is, if $\mathcal{E}$ is a trace-preserving quantum operation, then

$$
T(\mathcal{E}(\rho), \mathcal{E}(\sigma)) \leqslant T(\rho, \sigma)
$$

for all density matrices, $\rho$ and $\sigma$. For our purposes, it is especially important to note that this is true for the case where $\mathcal{E}$ is a partial trace operation, as the partial trace is a trace-preserving quantum operation.

Fannes $[14,15]$ proved a useful continuity relation relating trace distance and entropy. Fannes' inequality states that for any density matrices $\rho$ and $\sigma$ such that $T(\rho, \sigma) \leqslant 1 / e$,

$$
|S(\rho)-S(\sigma)| \leqslant T(\rho, \sigma) \log _{2}(d)+\eta(T(\rho, \sigma)),
$$

where $d$ is the dimension of the Hilbert space, $\rho$ and $\sigma$ are defined on $\eta(x) \equiv-x \log _{2}(x)$, and the base of logarithms, here and throughout, is taken to be 2 . It is useful to note that $\eta(x)$ is increasing for $0 \leqslant x \leqslant 1 / e$. The restriction on Eq. (2) that $T(\rho, \sigma) \leqslant 1 / e$ may be lifted to give

$$
|S(\rho)-S(\sigma)| \leqslant T(\rho, \sigma) \log _{2}(d)+\frac{\log _{2}(e)}{e} .
$$

Ruskai's result [Eq. (1)] can be combined with Fannes' inequality $[\mathrm{Eq} .(2)]$ to obtain the desired continuity relation for pure state entanglement. Suppose $|\psi\rangle$ and $|\phi\rangle$ are pure 
states of a composite quantum system with components $A$ and $B$, and that system $A$ has dimension $d$. Let $\rho_{A}$ and $\sigma_{A}$ be the corresponding reduced density matrices for system $A$. Applying Fannes's inequality [Eq. (2)] gives

$$
\begin{aligned}
|E(\psi)-E(\phi)| & =\left|S\left(\rho_{A}\right)-S\left(\sigma_{A}\right)\right| \\
& \leqslant T\left(\rho_{A}, \sigma_{A}\right) \log _{2}(d)+\eta\left(T\left(\rho_{A}, \sigma_{A}\right)\right) .
\end{aligned}
$$

Recalling that $\eta(x)$ is monotonically increasing for $0 \leqslant x$ $\leqslant 1 / e$, and using Eq. (1) to deduce that $T\left(\rho_{A}, \sigma_{A}\right)$ $\leqslant T(\psi, \phi)$, we obtain

$$
|E(\psi)-E(\phi)| \leqslant T(\psi, \phi) \log _{2} d+\eta(T(\psi, \phi)),
$$

provided $T(\psi, \phi) \leqslant 1 / e$. This is the desired continuity relationship for the pure-state entanglement. Using Eq. (3) we may lift the restriction $T(\psi, \phi) \leqslant 1 / e$ to give the bound

$$
|E(\psi)-E(\phi)| \leqslant T(\psi, \phi) \log _{2} d+\frac{\log _{2}(e)}{e} .
$$

We now generalize these pure-state results to apply to the entanglement of formation. Our strategy for proving a continuity bound for the entanglement of formation involves three ingredients in addition to those used in the proof of the bound for the pure-state entanglement [Eq. (6)]: Uhlmann's formula for the fidelity [16], the Bures distance [17], and the remote-control view of entanglement [18].

First, however, we must define the entanglement of formation. For a density matrix $\rho$ of a composite system $A B$ the entanglement of formation is defined by [10]

$$
E(\rho) \equiv \min \sum_{m} p_{m} S\left(\rho_{A, m}\right)
$$

where the minimization is over all ensembles $\left\{p_{m},\left|A B_{m}\right\rangle\right\}$ generating the state $\rho$, that is, $\rho=\Sigma_{m} p_{m}\left|A B_{m}\right\rangle\left\langle A B_{m}\right|$, and $\rho_{A, m} \equiv \operatorname{tr}_{B}\left(\left|A B_{m}\right\rangle\left\langle A B_{m}\right|\right)$. In practice, evaluating this expression seems to be very difficult; all that is known is an ingenious expression for the entanglement of formation of a pair of qubits found by Wootters [10], building on earlier work by Hill and Wootters [9].

The first ingredient needed to prove the continuity bound for the entanglement of formation is the fidelity, a measure of distance between two density matrices distinct from but closely related to the trace distance. The fidelity between density matrices $\rho$ and $\sigma$ is defined to be $F(\rho, \sigma)$ $\equiv \operatorname{tr} \sqrt{\rho^{1 / 2} \sigma \rho^{1 / 2}}$. For pure states $|\psi\rangle$ and $|\phi\rangle$ the fidelity reduces to the overlap between the states, $F(\psi, \phi)=|\langle\psi \mid \phi\rangle|$. The fidelity is not a metric; however, it does possess many useful properties as a measure of distance, and is closely related to the trace distance and will be used in the definition of the Bures distance [19-21]. Uhlmann [16,22] found a useful expression for the fidelity relying on the following construction. Suppose $\rho$ and $\sigma$ are quantum states of a $d$-dimensional quantum system. We label the system $Q$ for convenience. Introduce an additional "reference" system $R$
[23] of any fixed dimensionality that is at least as great as $d$. Uhlmann's expression for the fidelity is

$$
F(\rho, \sigma)=\max |\langle\rho \mid \sigma\rangle|=\max F(|\rho\rangle,|\sigma\rangle),
$$

where the maximization is performed over all pure states $|\rho\rangle$ and $|\sigma\rangle$ of the joint system $R Q$ such that $\operatorname{tr}_{R}(|\rho\rangle\langle\rho|)=\rho$ and $\operatorname{tr}_{R}(|\sigma\rangle\langle\sigma|)=\sigma$; such states are known as purifications of $\rho$ and $\sigma$.

As our second ingredient, we introduce the Bures distance [17] between density matrices $\rho$ and $\sigma$ :

$$
D(\rho, \sigma) \equiv 2 \sqrt{1-F(\rho, \sigma)} .
$$

$D(\cdot, \cdot)$ is easily shown to be a metric on the space of density matrices. We have chosen the overall normalization factor of 2 out the front so the Bures distance $D(\cdot, \cdot)$ agrees with the trace distance for pure states; other authors often use different normalizations.

The third ingredient we need is the elegant remote-control view of entanglement [18]. Suppose $\rho$ is some joint state of a composite system $A B$, where $A$ is $d$-dimensional and $B$ is $d^{\prime}$-dimensional. Uhlmann [24] showed that it is possible to achieve this minimum using an ensemble containing at most $d^{2} d^{\prime 2}$ ensemble elements. Introduce a $d^{2} d^{\prime 2}$-dimensional reference system $R$ which purifies those systems into a pure state $|\rho\rangle$. Let $\left\{p_{m},\left|A B_{m}\right\rangle\right\}$ be the ensemble of states achieving the minimum in Eq. (8). A result of Hughston, Jozsa, and Wootters [25] implies that by performing a measurement on $R$ with respect to an appropriate orthonormal basis $|m\rangle$, the corresponding posterior states of $A B$ will be $\left|A B_{m}\right\rangle$, with probability $p_{m}$. Elementary calculation shows that after the measurement we have $S\left(R^{\prime}\right)=H\left(p_{m}\right)$ and $S\left(A R^{\prime}\right)$ $=H\left(p_{m}\right)+E(\rho)$, where $S\left(R^{\prime}\right)$ denotes the von Neumann entropy of $R$ after the measurement, and similarly for $S\left(A R^{\prime}\right) . H(\cdot)$ is the Shannon entropy function. Combining these observations results in the very useful expression

$$
E(\rho)=S\left(A R^{\prime}\right)-S\left(R^{\prime}\right) .
$$

If instead a measurement had been performed in some other orthonormal basis $\left|m^{\prime}\right\rangle$, then we would have had

$$
E(\rho) \leqslant S\left(A R^{\prime}\right)-S\left(R^{\prime}\right) .
$$

Let us now proceed to the proof of the continuity relation for the entanglement of formation. Let $\rho_{A B}$ and $\sigma_{A B}$ be two density matrices of the system $A B$, where $A$ has $d$ dimensions, and $B$ has $d^{\prime}$ dimensions. Introduce a $d^{2} d^{\prime 2}$-dimensional reference system $R$. By Uhlmann's formula [Eq. (9)] there exist purifications $|\rho\rangle$ and $|\sigma\rangle$ of $\rho_{A B}$ and $\sigma_{A B}$ to the system $A B R$ such that

$$
F\left(\rho_{A B}, \sigma_{A B}\right)=F(|\rho\rangle,|\sigma\rangle) .
$$

Suppose we measure system $R$ in a basis chosen such that $E\left(\sigma_{A B}\right)=S\left(\sigma_{A R}^{\prime}\right)-S\left(\sigma_{R}^{\prime}\right)$, where the primes denote density matrices after the measurement, and the initial state was $|\sigma\rangle$. Performing the same measurement with initial state $|\rho\rangle$ we see from Eq. (12) that $E\left(\rho_{A B}\right) \leqslant S\left(\rho_{A R}^{\prime}\right)-S\left(\rho_{R}^{\prime}\right)$. Taking the difference of these equations yields 


$$
E\left(\rho_{A B}\right)-E\left(\sigma_{A B}\right) \leqslant S\left(\rho_{A R}^{\prime}\right)-S\left(\sigma_{A R}^{\prime}\right)+S\left(\sigma_{R}^{\prime}\right)-S\left(\rho_{R}^{\prime}\right) .
$$

Applying Fannes' inequality [Eq. (2)] twice on the righthand side gives

$$
\begin{aligned}
E\left(\rho_{A B}\right)-E\left(\sigma_{A B}\right) \leqslant & \log _{2}\left(d^{3} d^{\prime 2}\right) T\left(\rho_{A R}^{\prime}, \sigma_{A R}^{\prime}\right) \\
& +\eta\left(T\left(\rho_{A R}^{\prime}, \sigma_{A R}^{\prime}\right)\right) \\
& +\log _{2}\left(d^{2} d^{\prime 2}\right) T\left(\rho_{R}^{\prime}, \sigma_{R}^{\prime}\right)+\eta\left(T\left(\rho_{R}^{\prime}, \sigma_{R}^{\prime}\right)\right) .
\end{aligned}
$$

By Eq. (1), we have

$$
T\left(\rho_{R}^{\prime}, \sigma_{R}^{\prime}\right) \leqslant T\left(\rho_{A R}^{\prime}, \sigma_{A R}^{\prime}\right) \leqslant T\left(\rho_{A B R}^{\prime}, \sigma_{A B R}^{\prime}\right) \leqslant T(|\rho\rangle,|\sigma\rangle) .
$$

Recall that $T(|\rho\rangle,|\sigma\rangle)=D(|\rho\rangle,|\sigma\rangle)$. Together with the previous equation this fact and Eq. (13) give

$$
T\left(\rho_{R}^{\prime}, \sigma_{R}^{\prime}\right) \leqslant T\left(\rho_{A R}^{\prime}, \sigma_{A R}^{\prime}\right) \leqslant D\left(\rho_{A B}, \sigma_{A B}\right) .
$$

Combining this equation with Eq. (15) gives

$$
\begin{aligned}
E\left(\rho_{A B}\right)-E\left(\sigma_{A B}\right) \leqslant & {\left[5 \log _{2}(d)+4 \log _{2}\left(d^{\prime}\right)\right] D\left(\rho_{A B}, \sigma_{A B}\right) } \\
& +2 \eta\left(D\left(\rho_{A B}, \sigma_{A B}\right)\right)
\end{aligned}
$$

provided $D\left(\rho_{A B}, \sigma_{A B}\right) \leqslant 1 / e$. This is the desired continuity equation for the entanglement of formation. Of course, the role of $A$ and $B$ may be interchanged in this expression; clearly the strongest inequality is obtained by labeling the systems such that $d \leqslant d^{\prime}$. For many purposes it is sufficient to replace the logarithmic terms in the right-hand side by $9 \log _{2}\left[\max \left(d, d^{\prime}\right)\right]$.

The restriction $D\left(\rho_{A B}, \sigma_{A B}\right) \leqslant 1 / e$ on Eq. (18) may be lifted in a manner similar to that for the continuity bound for pure-state entanglement. Doing so gives

$$
\begin{aligned}
E\left(\rho_{A B}\right)-E\left(\sigma_{A B}\right) \leqslant & {\left[5 \log _{2}(d)+4 \log _{2}\left(d^{\prime}\right)\right] D\left(\rho_{A B}, \sigma_{A B}\right) } \\
& +2 \log _{2}(e) / e .
\end{aligned}
$$

For applications to communication in which large blocks of entanglement are used and $d$ becomes large, it is desirable to understand how close to optimal (with respect to $d$ ) the bounds (18) and (19) are. Understanding this is essentially the problem of understanding how close to optimal Fannes' inequality is. Let $\epsilon>0$ be given, and, for a $d$-dimensional Hilbert space with orthonormal basis $|1\rangle, \ldots,|d\rangle$ define $\rho$ $\equiv \epsilon d|1\rangle\langle 1|+(1 / d-\epsilon) I$. For small $\epsilon$ this is a density matrix close to the completely mixed state $I / d$. We will analyze the difference in entropies between $\rho$ and $I / d$. From the general bound [26] $S\left(\Sigma_{i} p_{i} \rho_{i}\right) \leqslant H\left(p_{i}\right)+\Sigma_{i} p_{i} S\left(\rho_{i}\right)$ we obtain

$$
S(\rho) \leqslant(1-\epsilon d) \log _{2}(d)+H(\epsilon d, 1-\epsilon d) .
$$

Thus

$$
\begin{aligned}
S(I / d)-S(\rho) & \geqslant \epsilon d \log _{2}(d)-H(\epsilon d, 1-\epsilon d) \\
& \geqslant \epsilon d \log _{2}(d)-1 .
\end{aligned}
$$

A simple calculation shows that $T(I / d, \rho)=2(d-1) \epsilon$. It follows easily that

$$
S(I / d)-S(\rho) \geqslant \frac{T(I / d, \rho) \log _{2}(d)}{2}-1 .
$$

This implies that the logarithmic behavior (with $d$ ) expressed in Eq. (2) and thus in Eqs. (6), (7), (18), and (19) cannot be improved beyond a constant factor.

Vidal [7] emphasized the importance of continuity to the result of Popescu and Rohrlich [6] (see also Ref. [12]). Popescu and Rohrlich argued that any measure of bipartite purestate entanglement satisfying certain natural axioms, namely, that it is (a) additive, and (b) nonincreasing under local operations and classical communication, is necessarily proportional to the von Neumann entropy of the reduced density matrix of the pure state. Vidal pointed out some hidden assumptions in this argument by explicitly constructing examples of entanglement measures that satisfy (a) and (b), yet are not proportional to the von Neumann entropy. For example, a function with the required properties is $\widetilde{E}(\psi) \equiv$ $-\log _{2}\left[\operatorname{tr}\left(\rho^{2}\right)\right]$, which is manifestly different from the von Neumann entropy. Vidal pointed out that the key property lacking in such a measure is sufficiently strong continuity properties. The framework of the present note provides a useful opportunity to elaborate. Suppose $\widetilde{E}(\rho)$ is any additive measure of entanglement that does not increase under local operations and classical communication. We will show that $\widetilde{E}(\rho)$ cannot satisfy a continuity property as strong as Eq. (18) unless for pure states it is proportional to the von Neumann entropy. Indeed for any constants $C$ and $D$ we will show that a continuity property as strong as

$$
|\widetilde{E}(\rho)-\widetilde{E}(\sigma)| \leqslant C \log _{2}(d) D(\rho, \sigma)+D
$$

implies the $\widetilde{E}$ is proportional to the von Neumann entropy of the reduced density matrix, where $d$ is the maximum of the dimensions of systems $A$ and $B$. Thus the von Neumann entropy is in some sense the "most continuous" measure of entanglement, satisfying a stronger bound on its variation than any other prospective measure of entanglement. Suppose Eq. (24) holds. Let $\epsilon>0$ be given. Then for sufficiently large $n$ entanglement dilution [27] allows us to convert from $n[S(\rho)+\epsilon]$ Bell states into a state $\sigma$ that satisfies $D\left(|\psi\rangle^{\otimes n}, \sigma\right) \leqslant \epsilon$, using local operations and classical communication. Then, if Eq. (24) holds,

$$
n \widetilde{E}(\psi)=\widetilde{E}\left(\psi^{\otimes n}\right) \leqslant \widetilde{E}(\sigma)+C \log _{2}\left(d^{n}\right) \epsilon+D,
$$

since $D\left(|\psi\rangle^{\otimes n}, \sigma\right) \leqslant \epsilon$. The nonincrease of $\widetilde{E}$ under local operations and classical communication implies that $\widetilde{E}(\sigma)$ $\leqslant n[S(\rho)+\epsilon] k$, where $k$ is the entanglement associated with a single Bell pair according to the measure $\widetilde{E}$. Thus

$$
n \widetilde{E}(\psi) \leqslant n(S(\rho)+\epsilon) k+C n \log _{2}(d) \epsilon+D .
$$

Dividing by $n$ and letting $\epsilon \rightarrow 0, n \rightarrow \infty$ gives $\widetilde{E}(\psi) \leqslant k S(\rho)$. Similarly, for any $\epsilon>0$ and sufficiently large $n$, entangle- 
ment concentration [27] allows us to convert $n$ copies of $|\psi\rangle$ into a state $\tau$ satisfying $D\left(|\beta\rangle^{\otimes n(S(\rho)-\epsilon)}, \tau\right)<\epsilon$, where $|\beta\rangle$ is a Bell state. By Eq. (24) we have

$$
n[S(\rho)-\epsilon] k \leqslant \widetilde{E}(\tau)+C n \log _{2}(d) \epsilon+D .
$$

But $\widetilde{E}(\tau) \leqslant n \widetilde{E}(\psi)$, since $\widetilde{E}$ is nonincreasing under local operations and classical communication. Thus

$$
\begin{aligned}
n[S(\rho)-\epsilon] k & =\widetilde{E}\left(\beta^{\otimes n(S(\rho)-\epsilon)}\right) \\
& \leqslant n \widetilde{E}(\psi)+C n \log _{2}(d) \epsilon+D .
\end{aligned}
$$

Dividing by $n$ and letting $\epsilon \rightarrow 0, n \rightarrow \infty$ gives $k S(\rho) \leqslant \widetilde{E}(\psi)$.

Combining the results of the last paragraph, we see that the properties of being additive, nonincreasing under local operations and classical communication, and satisfying Eq. (24) for some $C$ and $D$, imply that $\widetilde{E}(\psi)=k S(\rho)$. Thus measures of pure state entanglement such as $\widetilde{E}(\psi)=$ $-\log _{2}\left(\operatorname{tr}\left(\rho^{2}\right)\right)$ which are not proportional to $S(\rho)$ must satisfy weaker continuity relations than Eq. (24).

Finally, it should be mentioned that bounds (18) and (19) apply only to the entanglement of formation as defined in Eq. (8). As discussed by Wootters [10], the interpretation of the entanglement of formation as defined in Eq. (8) may be somewhat problematic. The basic problem is that one would like to interpret the entanglement of formation as a measure of the resources-Bell pairs-that must be shared between Alice and Bob in order to create $\rho$. That is, if Alice and Bob are provided with $n E(\rho)$ Bell pairs, then by local operations and classical communication they can convert them with high fidelity into $n$ copies of $\rho$, in the limit that $n$ is large. As Wootters discussed, the only impediment to this interpretation is the question of whether or not the entanglement of formation is additive. That is, is it true that $E\left(\rho^{\otimes n}\right)$ $=n E(\rho)$ ? If this is not true, then it suggests a revised definition for the entanglement of formation, the operational entanglement of formation, as $E_{o p}(\rho) \equiv \lim _{\sup } \operatorname{sum}_{n \rightarrow \infty} E\left(\rho^{\otimes n}\right) / n$. $E_{o p}(\rho)$ quantifies the resources needed for Alice and Bob to create $\rho$, in the sense described above. Unfortunately, the reasoning used in the derivation of the continuity bounds on the entanglement of formation (18) and (19) does not go through for $E_{o p}(\rho)$. It is an interesting open problem to determine such bounds for the operational entanglement of formation, and may yield insight into the question of whether or not the entanglement of formation is additive.

We have obtained a continuity relation for bipartite purestate entanglement and the entanglement of formation. This relation bounds the variation of the entanglement $E(\rho)$ between two systems $A$ and $B$ as the state of the joint system $\rho$ is varied. The bound obtained exhibits the best possible behavior with respect to the dimension $d$ of the underlying Hilbert space, to within constant factors, and is stronger than the continuity bounds that may be obtained for other potential measures of entanglement. Further applications to quantum communication protocols, quantum cloning, and quantum communication complexity will be reported elsewhere.

This research was supported by DARPA through the Quantum Information and Computing Institute (QUIC) administered through the ARO, and by the California Institute of Technology.
[1] A. Peres, Quantum Theory: Concepts and Methods (Kluwer, Dordrecht, 1993).

[2] P. W. Shor, SIAM J. Comput. 26, 1484 (1997).

[3] L. K. Grover, Phys. Rev. Lett. 79, 325 (1997).

[4] C. H. Bennett et al., Phys. Rev. Lett. 70, 1895 (1993).

[5] W. H. Zurek, Phys. Today 44 (10), 36 (1991).

[6] S. Popescu and D. Rohrlich, Phys. Rev. A 56, R3319 (1997).

[7] G. Vidal, e-print quant-ph/9807077.

[8] C. H. Bennett, D. P. DiVincenzo, J. A. Smolin, and W. K. Wootters, Phys. Rev. A 54, 3824 (1996).

[9] S. Hill and W. K. Wootters, Phys. Rev. Lett. 78, 5022 (1997).

[10] W. K. Wootters, Phys. Rev. Lett. 80, 2245 (1998).

[11] V. Vedral and M. B. Plenio, Phys. Rev. A 57, 1619 (1998).

[12] P. Horodecki, M. Horodecki, and R. Horodecki, Phys. Rev. Lett. 82, 1056 (1999).

[13] M. B. Ruskai, Rev. Math. Phys. 6, 1147 (1994).

[14] M. Fannes, Commun. Math. Phys. 31, 291 (1973).
[15] M. Ohya and D. Petz, Quantum Entropy and its Use (SpringerVerlag, Berlin, 1993).

[16] A. Uhlmann, Rep. Math. Phys. 9, 273 (1976).

[17] D. Bures, Trans. Am. Math. Soc. 135, 199 (1969).

[18] D. P. DiVincenzo et al., LANL e-print, quant-ph/9803033.

[19] C. A. Fuchs and J. van de Graaf, IEEE Trans. Inf. Theory 45, 1216 (1999).

[20] C. A. Fuchs, Ph.D. thesis, University of New Mexico, Albuquerque, NM, 1996; also LANL e-print quant-ph/9601020.

[21] M. A. Nielsen, Ph.D. thesis, University of New Mexico, 1998.

[22] R. Jozsa, J. Mod. Opt. 41, 2315 (1994).

[23] B. W. Schumacher, Phys. Rev. A 54, 2614 (1996).

[24] A. Uhlmann, e-print quant-ph/9704017.

[25] L. P. Hughston, R. Jozsa, and W. K. Wootters, Phys. Lett. A 183, 14 (1993).

[26] A. Wehrl, Rev. Mod. Phys. 50, 221 (1978).

[27] C. H. Bennett, H. J. Bernstein, S. Popescu, and B. Schumacher, Phys. Rev. A 53, 2046 (1996). 